\title{
Severity of depression and risk for subsequent dementia: cohort studies in China and the UK*
}

Ruoling Chen, Zhi Hu, Li Wei, Xia Qin, Cherie McCracken and John R. Copeland

\section{Background}

Depression and dementia often exist concurrently. The associations of depressive syndromes and severity of depression with incident dementia have been little studied.

\section{Aims}

To determine the effects of depressive syndromes and cases of depression on the risk of incident dementia.

\section{Method}

Participants in China and the UK aged $\geqslant 65$ years without dementia were interviewed using the Geriatric Mental State interview and re-interviewed 1 year later in 1254 Chinese, and 2 and 4 years later in 3341 and 2157 British participants respectively (Ageing in Liverpool Project Health Aspects: part of the Medical Research Council - Cognitive Function and Ageing study).

\section{Results}

Incident dementia was associated with only the most severe depressive syndromes in both Chinese and British participants. The risk of dementia increased, not in the less severe cases of depression but in the most severe cases. The multiple adjusted hazard ratio $(\mathrm{HR})=5.44(95 \%$ $\mathrm{Cl}$ 1.67-17.8) for Chinese participants at 1-year follow-up, and $\mathrm{HR}=2.47(95 \% \mathrm{Cl} 1.25-4.89)$ and $\mathrm{HR}=2.62(95 \%$ Cl 1.18-5.80) for British participants at 2- and 4-year follow-up respectively. The effect was greater in younger participants.

\section{Conclusions}

Only the most severe syndromes and cases of depression are a risk factor for dementia.

\section{Declaration of interest}

None. Funding detailed in Acknowledgements.
Depression and dementia are the most prevalent and disabling mental disorders among older populations. It has been predicted that the number of people with dementia in the world will double every 20 years to 42 million by 2020 as the aged population grows. ${ }^{1}$ Evidence that depression and dementia often exist concurrently has stimulated speculation that there are complex associations between these two conditions. ${ }^{2}$ Many studies, ${ }^{3,4}$ but not all, ${ }^{5}$ have reported that a history of depression is a risk factor for dementia. However, recent data ${ }^{6,7}$ have shown that depressive symptoms are cross-sectionally associated with cognitive impairment but not with subsequent cognitive decline, suggesting that the presence of depression alone does not increase the risk of cognitive decline. This may raise questions about the risk of dementia in relation to depression. Particularly, we could find no investigation linking depressive syndromes, as opposed to depressive symptoms with increased risk of dementia.

Previous studies on the association of depression with dementia have mainly been carried out in the Western world. Also, few investigators have examined the effects of the severity of depression on dementia. ${ }^{3,8}$ In many Western populations, low socio-economic status, low social support, high cardiovascular risk factors and depression tend to co-occur, ${ }^{9-11}$ making a relationship between depression and dementia difficult to unravel. By contrast with Western populations, older people in China exhibit different patterns of risk factors with extremes of absolute deprivation combined with high levels of social supports, ${ }^{12}$ low levels of depression ${ }^{13,14}$ and low levels of some cardiovascular risk factors (e.g. serum cholesterol, body mass index). ${ }^{15,16}$ Studying such a population may therefore, offer internationally applicable insights into the aetiological role of depression in dementia.

*An abstract of this study was presented at the 135th Annual Meeting of the American Public Health Association, Washington DC, USA, 3-6 November 2007.
Using a semi-standardised method, the Geriatric Mental State (GMS) ${ }^{17}$ we examined the mental status of older residents in China, ${ }^{14}$ following our Medical Research Council - Ageing in Liverpool Project Health Aspects (MRC-ALPHA) study in the UK. ${ }^{10}$ The participants were diagnosed as having both different levels of syndromes and cases of depression and dementia. In this study we analysed data from two cohorts, one in China and the other in the UK, to investigate whether there were: any 'dose-response' associations for depressive syndromes and cases, with incident dementia; and possible variations with age, gender, ethnicity, cardiovascular disease (CVD) comorbidity, and type of depression.

\section{Method}

\section{Participants}

Chinese participants were those from our Hefei cohort study in Anhui Province, China. The methods used in the study have been fully described elsewhere. ${ }^{14}$ Briefly, in 2001 we randomly selected 1810 people aged $\geqslant 65$ years from the residency committee lists who had lived for at least 5 years in Yiming district of Hefei city, Anhui. Permission for interview and informed consent were obtained from each perosn but if that was not possible, from the closest responsible adult. Refusals were respected. Ethical approval was obtained from Anhui Medical University and the district government.

There were 1736 people who agreed to participate in the study (a response rate of 95.9\%). Participants were interviewed at home by a trained survey team from the School of Health Administration, Anhui Medical University. The main interview materials were the GMS - a comprehensive semi-structured mental state interview ${ }^{17}$ and a general health record that included risk factors. ${ }^{14}$ Blood pressure and physical measurements 
were taken. The validation study of depression cases was carried out by two consultant psychiatrists. A year after the baseline interview, 1293 participants (74.5\%) were successfully re-investigated, using the same protocol.

British participants were those from the MRC-ALPHA study, ${ }^{10}$ part of the MRC Cognitive Function and Ageing Study (www.cfas. ac.uk). The study methods have been fully described elsewhere. ${ }^{18}$ In brief, a sample of 6035 older people aged $\geqslant 65$ were randomly selected using the Liverpool Family Practitioner Committee central computerised list of general practice patients in 1989. Of these, 5222 participants were interviewed with a response rate of $86.5 \%$. The main interview materials included the GMS and the Minimum Data Set. ${ }^{19}$ Two years later, 3519 $(67.4 \%)$ participants were successfully re-interviewed, and 4 years later 2238 (42.9\%) participants were re-investigated. Ethical approval was obtained from the multicentre and local ethical committee.

\section{Assessment of syndromes and cases of depression and dementia}

A computer program assisted diagnosis - the Automated Geriatric Examination for Computer Assisted Taxonomy (AGECAT) - and was used to analyse the information from the GMS to identify the principal mental disorders in participants. ${ }^{17,20}$ It was developed using a theoretical model and tested against its success at replicating diagnoses on samples diagnosed by psychiatrists. It attempts to replicate the process by which a psychiatrist achieves a syndromal diagnosis followed by a differential diagnosis. Geriatric Mental State symptoms are coalesced into a 150 'symptoms components'. In stage I the symptom components are brought together into groups which typify the major symptom areas of each diagnostic syndrome. The scores on these individual groups determine the final syndromal level of 'confidence of diagnosis'. Thus, the system uses both quantitative and qualitative measures when allocating participants to the levels of confidence; required for its construction are many hundreds of clinical decisions on the placement of groups of symptom components on the syndrome levels. Individual participants are allocated to levels of confidence of diagnosis (0-5) on each of the eight diagnostic syndromes: organic disorder, depression, mania, schizophrenia and paranoid, obsessional, phobic, hypochondriacal, and general anxiety. In stage II the various syndrome levels are compared to derive a final differential diagnosis, a level of confidence of diagnosis from $0-5$. A level of $\geqslant 3$ in most circumstances designates a 'case level' that has been shown to correspond with what psychiatrists usually recognise as a case for intervention. Levels 1 and 2 are designated as 'sub-cases', and level 0 (no confidence level on any syndrome) is classified as 'well'. ${ }^{21}$ Although the levels are intended to be levels of confidence of diagnosis, it has been our observation that, in those conditions where diagnosis tends to be on a continuum with normality, for example in cognitive decline and depression, the different levels tend also to reflect levels of severity in terms of type and numbers of symptoms. Here we use 'severity' in terms of the symptoms themselves not in terms of their impact on daily living. In community-based samples the proportion of organic cases not resulting from dementia is small; acute confusional states have been estimated in about $0.5 \%$ of the community sample and the proportion of participants with depression sufficiently severe to be diagnosed as an organic state at case level also appear to be small. It has been the practice in community studies to ignore them.

A number of studies comparing AGECAT diagnoses for depression and dementia with psychiatrists' diagnoses using DSM-III and DSM-IV or ICD-10 criteria achieved a good level of agreement, ${ }^{20}$ including in older Chinese people. ${ }^{22,23}$ The AGECAT also divides depression into psychotic and neurotic types that appear to equate well with $\mathrm{DSM}-\mathrm{III}^{24} / \mathrm{DSM}-\mathrm{IV}^{25}$ major affective disorder and dysthymia without the 2-year rule. 'Type of depression' refers to diagnostic cases which emerge after stage II where syndrome diagnoses have been converted into a single differential diagnosis. The symptoms defining depression and dementia are not similar. The GMS-AGECAT diagnosis has been the most widely used international community-based study method for investigations of depression and dementia in older people. $^{17}$

\section{Statistical analysis}

Data were analysed for those who did not have dementia at baseline and were followed up (1254 Chinese and 3341 British). The associations of baseline depressive syndromes with subsequent dementia at follow-up were explored using a Cox regression model with adjustment for age, gender, educational level and CVD. In examining the risk of dementia in relation to depression, baseline sub-cases of dementia were further allowed in the multiple regression model for analysis. Differences in the relationship between subgroups of age, gender, ethnicity, CVD comorbidity and type of depression were tested. ${ }^{26}$ All analyses were performed in SPSS version 13.0 for Windows.

\section{Results}

Table 1 shows number and incidence of dementia across baseline depressive syndromes. There were no level 5 depressive syndromes (nor cases) in our community-based samples. The risk of dementia was not associated with level 1-3 depressive syndromes, but significantly increased with level 4 . Differences in the increased hazard ratio (HR) between Chinese and British participants were not significant $(P>0.20)$. This relationship was further observed with baseline depression cases at level 4 only, but not with less severe cases at level 3 and sub-cases at levels 1-2. These findings remained significant even after further consideration of baseline dementia subcases, which were associated with incident dementia (Fig. 1).

We investigated differences in baseline covariables between MRC-ALPHA participants with depression at level $4 \quad(n=108)$ and those at level $3(n=375)$. There were no significant differences with age (77.9 v. 77.6), gender (male $40.7 \%$ v. 38.9\%), education years (9.0 v. 9.2), Townsend score - an index for socio-economic deprivation $^{27}-(6.14$ v. 5.56), treated hypertension $(26.9 \% v$. $30.7 \%)$, heart attack (9.3\% v. $13.6 \%)$, angina/chest pain $(15.7 \%$ v. $17.9 \%)$, and stroke/parts of body paralysed (11.1\% v. 11.7\%). However, individuals with level 4 depression had more organic syndromes at level $\geqslant 3(8.3 \%$ v. $0.8 \%, P<0.001)$, more depressive neurosis $(62.0 \%$ v. $21.9 \%, P<0.001)$ and more antidepressant drug use ${ }^{28}$ (benzodiazepines $37.0 \%$ v. $21.6 \%, P=0.001$; tricyclic antidepressants, selective serotonin reuptake inhibitors, lofepramine and others $17.6 \%$ v. $8.0 \%, P=0.004)$. After further adjustment for these medications, the results were not substantially changed; multiple adjusted HR for incident dementia was 2.41 (95\% CI 1.20-4.85) and $2.54(1.13-5.69)$ in the 2- and 4-year follow-up respectively.

We examined differences in the effects of level 4 depression on incident dementia between the subgroups in the MRC-ALPHA combined data of incident dementia in the 4-year follow-up with a 2-year follow-up (i.e. adding the Wave III incident dementia into Wave II). There were no significant differences in the adjusted HRs between men (HR=1.73, 95\% CI 0.61-4.91) v. women $(\mathrm{HR}=2.07,95 \%$ CI $1.03-4.15) \quad(P=0.78)$; people with CVD 


\begin{tabular}{|c|c|c|c|c|c|c|}
\hline \multirow[b]{2}{*}{ Depressive syndrome at baseline } & \multicolumn{2}{|c|}{$\begin{array}{l}\text { Chinese participants } \\
\text { (1-year follow-up) }\end{array}$} & \multicolumn{2}{|c|}{$\begin{array}{l}\text { British participants } \\
\text { (2-year follow-up) }\end{array}$} & \multicolumn{2}{|c|}{$\begin{array}{l}\text { British participants } \\
\text { (4-year follow-up) }\end{array}$} \\
\hline & $\begin{array}{l}\text { Incident dementia } \\
n / N(\%)\end{array}$ & $\begin{array}{l}\text { Adjusted } \mathrm{HR}^{\mathrm{a}} \\
\quad(95 \% \mathrm{Cl})\end{array}$ & $\begin{array}{l}\text { Incident dementia } \\
n / N(\%)\end{array}$ & $\begin{array}{l}\text { Adjusted } \mathrm{HR}^{\mathrm{a}} \\
\quad(95 \% \mathrm{Cl})\end{array}$ & $\begin{array}{l}\text { Incident dementia } \\
n / N(\%)\end{array}$ & $\begin{array}{l}\text { Adjusted } \mathrm{HR}^{\mathrm{a}} \\
(95 \% \mathrm{Cl})\end{array}$ \\
\hline Level $0^{\mathrm{b}}$ & 67/1178 (5.7) & 1.00 & 155/2601 (6.0) & 1.00 & 137/1720 (8.0) & 1.00 \\
\hline Level 1 & $4 / 24(16.7)$ & $\begin{array}{c}\text { Combined with } \\
\text { level } 2\end{array}$ & 17/204 (8.3) & $1.57(0.95-2.61)$ & 15/131 (11.5) & $1.34(0.77-2.34)$ \\
\hline Level 2 & 0/22 (0.0) & $1.15(0.41-3.21)$ & $12 / 225(5.3)$ & $0.79(0.44-1.43)$ & 7/125 (5.6) & $0.55(0.25-1.21)$ \\
\hline Level 3 & 1/26 (3.8) & $0.58(0.08-4.26)$ & $12 / 236(5.1)$ & $0.95(0.52-1.71)$ & 9/143 (6.3) & $0.85(0.43-1.68)$ \\
\hline Level 4 & 3/4 (75.0) & $5.05(1.56-16.3)^{\star *}$ & 10/75 (13.3) & $2.13(1.12-4.06)^{\star}$ & 8/38 (21.1) & $2.45(1.17-5.15)^{*}$ \\
\hline
\end{tabular}

comorbidities $(\mathrm{HR}=1.47,95 \%$ CI $0.44-4.86) v$. without CVD $(\mathrm{HR}=2.17,95 \%$ CI $1.12-4.22) \quad(P=0.58)$; and depressive neurosis $(\mathrm{HR}=2.77,95 \%$ CI 1.22-6.26) v. depressive psychosis $(\mathrm{HR}=1.66$, 95\% CI 0.78-3.53) ( $P=0.37)$. However, the effect increased with younger age; adjusted HRs in those aged $65-74,75-84$ and $85+$ years were 6.10 (95\% CI 1.92-19.4), 2.16 (95\% CI $0.92-5.08)$ and 1.05 (95\% CI $0.45-1.94)$ respectively; $P=0.012$ in the youngest $v$. the oldest.

We carried out two sensitivity analyses to investigate further the effects of baseline depression on incident dementia using the above combined data. First, after excluding all participants with baseline organic syndrome at level $\geqslant 3$, adjusted HRs in level 4 depression ( $n=10,15.4 \%$ incident dementia) was 1.89 (95\% CI 1.00-3.57), while those for depression at levels 1,2 and 3 were 1.36 (95\% CI $0.84-2.21), 0.85$ (95\% CI $0.52-1.40$ ) and 0.88 (95\% CI 0.53-1.44) respectively. Second, excluding 73 participants with incident dementia who at the same time had depressive syndrome at level $\geqslant 3$ from 330 individuals with incident dementia, the matched figures were 1.46 (95\% CI $0.68-3.10), 0.53$ (95\% CI 0.26-1.08), 0.55 (95\% CI 0.28-1.07) and 1.18 (95\% CI 0.66-2.13).

\section{Discussion}

In two large population-based cohorts in China and the UK, we found that an increased risk of dementia was associated with only the most severe syndromes and cases of depression, but not with less severe syndromes and cases. The increased risk was not statistically different with ethnicity, gender, cardiovascular disease comorbidity or type of depression, but was higher in younger participants.

Our findings may weigh more towards particular causal pathways. Depression may be associated with increased risk of dementia because depression is a reaction to perceived cognitive deficits, because depression is an early symptom of dementia (occurring before the syndrome becomes clinically definable), or because depression is actually a risk factor for dementia. The fact that subcase and less severe case syndrome levels were not associated with dementia in this study may support the third explanation more than the first two (which would be less likely to show specificity to a particular syndrome level).

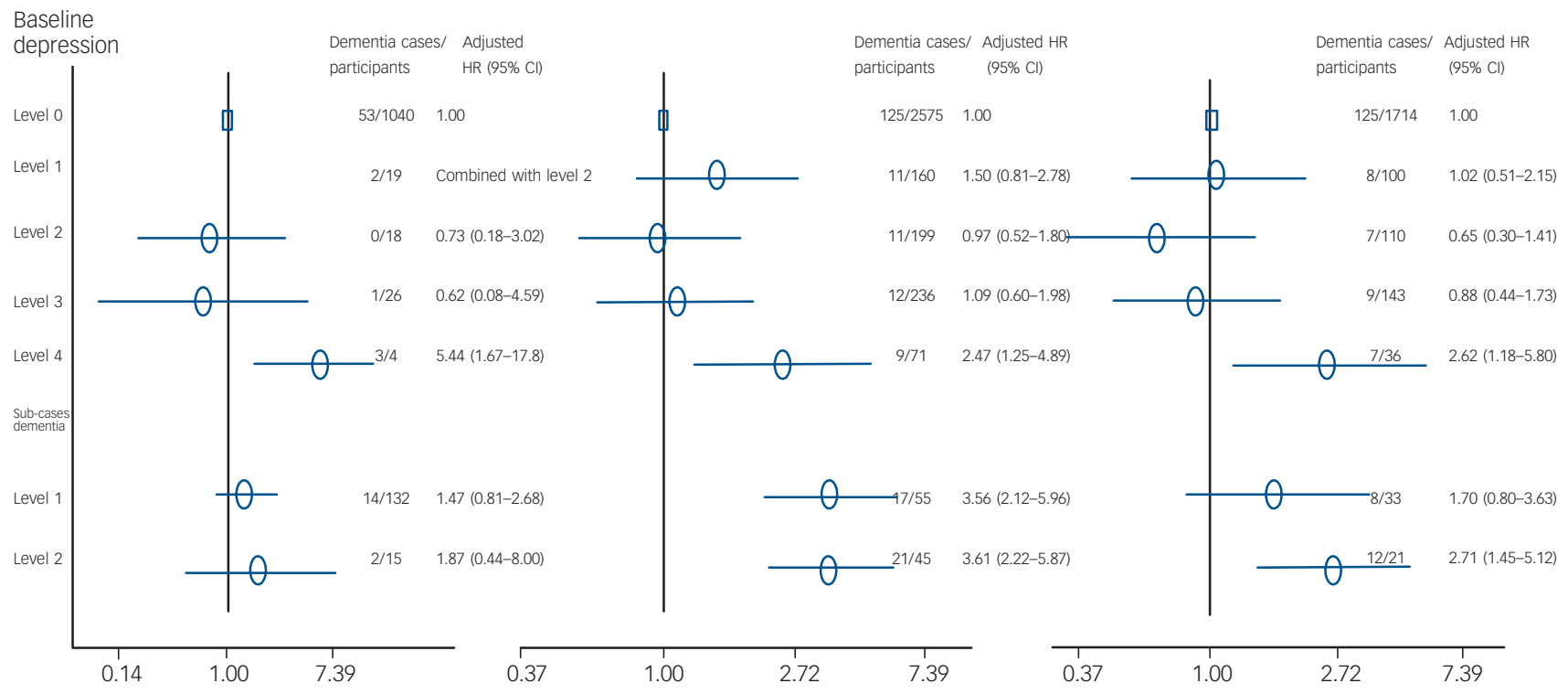

HR for dementia in 1-year follow-up - Chinese HR for dementia in 2-year follow-up - British

HR for dementia in 4-year follow-up - British 


\section{Strengths and limitations}

The strengths of the study were that we employed two markedly different populations in terms of levels of socio-economic status, social support, CVD and depression; the two cohorts had high response rates and the number of participants was relatively large, which gave sufficient power to test the relationship, including that in the subgroups; and the GMS-AGECAT, potentially measuring syndrome and diagnosis at 5 levels, offered a unique opportunity to examine dementia in relation to depressive severity (rather than simply as a dichotomous variable of depression).

The limitations of the study were that in the Chinese cohort, the incident dementia may be over-diagnosed because it included some illiterate participants ${ }^{14}$ (although the GMS-AGECAT was satisfactory for high-income countries with literate populations). ${ }^{29,30}$ The follow-up in the Chinese cohort was relatively short (1 year), and the numbers with depression and dementia were small (and thus it was not possible to examine differences in the baseline covariables between people with depression levels 3 and 4 , and differences in the effect of level 4 depression on dementia between the subgroups of participants). However, the findings were consistent and supported by the large British data with the long-term 4-year follow-up. Another limitation was that we could not separate dementia into Alzheimer's disease, vascular dementia, or both in order to examine their associations with depression. Thus, we could not directly link level 4 depression cases to Alzheimer's disease or vascular dementia. However, our data showed that depression at level 4 increased the risk of dementia in both participants with and without CVD, suggesting that the most severe cases of depression may be related to both vascular dementia and Alzheimer's disease. The finding that depression increased the risk of Alzheimer's disease has been shown in a recent meta-analysis paper, which did not examine the effects of the severity of depression on Alzheimer's disease. ${ }^{8}$

\section{Effects of depression on incident dementia}

To our knowledge, our study is the first to examine the relationship between depressive syndromes and the risk of dementia, and report that within clinical depressions, the less severe cases of depression did not increase any risk for developing dementia, but the most severe cases did so significantly. The MRC-ALPHA data showed that individuals with level 4 depression at baseline were more likely to have level 4 depression within 2 years than those with baseline level 3 (data on request), suggesting depression lasted longer at level 4 (although we cannot exclude some participants who may have recovered and then relapsed in the meantime). This longer duration may be a reflection of the level of depression severity. Several epidemiological studies have examined depression as a risk factor for dementia with conflicting results. ${ }^{4}$ In a meta-analysis, Jorm concluded that a history of depression increased the risk for developing dementia by $87 \%$. Our data suggest that the most severe level of depression increased the incidence of dementia to a greater degree than those in previous studies. ${ }^{3,8}$ We believe this is because we separated severe from less severe depression. This may explain the variable conclusions in those studies which grouped severity levels of depression together. Failure to confirm this finding may have been partly due to a lower proportion of severe depression in their samples.

Why does the GMS-AGECAT level 4, not case level 3 depression appear to increase the risk of incident dementia? Such a pattern even existed in our sensitivity analyses. It seems not to arise from differences in those covariables at baseline. It could not be entirely explained by the lower proportion of depressive neurosis (or higher depressive psychosis) in participants with level
3 depression at baseline compared with those with level 4, since level 4 depressive psychosis itself increased the risk of dementia. We considered that some symptoms or other unmeasured variables might have been included in level 4, but not in level 3, depression and/or simply the severity of depression could play a role. Perturbations of the hypothalamic-pituitary-adrenal axis in depression can lead to hypercortisolemia-associated hippocampal atrophy which may also explain why the most severe cases of depression are those more likely to increase the risk of dementia. Nevertheless, the GMS-AGECAT diagnosis, adapted for clinical use, could be used to help identify those at higher risk in older populations in an attempt to prevent dementia. The strong effect found in younger elderly people and the long-term effect may imply that depression at a younger age is more important for developing dementia, suggesting that early treatment of depression and prevention of depression in the younger population may effectively reduce dementia in later life.

The increased risk of incident dementia in relation to only the most severe syndromes or cases of depression is unlikely to result from chance or bias. Our study urges that the aetiological roles of the severity of depressive symptoms, syndromes and clinical cases on dementia need to be addressed. ${ }^{31}$ Early treatment and prevention of depression and relieving its severity could reduce the burden of dementia in both high-income and low- and middleincome countries.

Ruoling Chen, MD, PhD, Department of Epidemiology and Public Health, Royal Fre and University College Medical School, London, UK; Zhi Hu, MD, PhD, School of Health Administration, Anhui Medical University, China; Li Wei, MD, PhD, Medicines Monitoring Unit, Ninewells Hospital and Medical School, University of Dundee, UK; Xia Qin, BSc, School of Health Administration, Anhui Medical University, China; Cherie Mccracken, PhD, John R Copeland, MD, SCD, FRCP, Division of Psychiatry, University of Liverpool, UK.

Correspondence: Ruoling Chen, Department of Epidemiology and Public Health, University College London, 1-19 Torrington Place, London WC1E 6BT, UK. Email: ruoling.chen@ucl.ac.uk

First received 10 Sep 2007, final revision 4 Apr 2008, accepted 22 May 2008

\section{Acknowledgements}

We thank the many older Chinese and British residents who participated in the surveys, and the survey interviewers. Critical comments from two anonymous reviewers have substantially improved this paper. The Chinese cohort was funded by The Royal Society, UK (Grant NO. 574006.G603/22085) and the MRC-ALPHA cohort by the Medical Research Council (MRC) and later by MRC and the Department of Health, UK, as part of the MRCCFA Study. R.C. is supported by the BUPA Foundation. The opinions expressed in this report are not necessarily those of the funders.

\section{References}

1 Ferri $\mathrm{CP}$, Prince $\mathrm{M}$, Brayne $\mathrm{C}$, Brodaty $\mathrm{H}$, Fratiglioni L, Ganguli M, Hall K, Hasegawa $\mathrm{K}$, Hendrie $\mathrm{H}$, Huang $\mathrm{Y}$, Jorm A, Mathers $\mathrm{C}$, Menezes PR, Rimmer $\mathrm{E}$, Scazufca M; Alzheimer's Disease International. Global prevalence of dementia: a Delphi consensus study. Lancet 2005; 366: 2112-7.

2 Emery VO, Oxman TE. Update on the dementia spectrum of depression. Am J Psychiatry 1992; 149: 305-17.

3 Jorm AF. History of depression as a risk factor for dementia: an updated review. Aust N Z J Psychiatry 2001; 35: 776-81.

4 Andersen $\mathrm{K}$, Lolk A, Kragh-Sorensen $\mathrm{P}$, Petersen NE, Green A. Depression and the risk of Alzheimer disease. Epidemiology 2005; 16: 233-8.

5 Anonymous. The Canadian Study of Health and Aging: risk factors for Alzheimer's disease in Canada. Neurology 1994; 44: 2073-80.

6 Vinkers DJ, Gussekloo J, Stek ML, Westendorp RG, van der Mast RC. Temporal relation between depression and cognitive impairment in old age: prospective population based study. BMJ 2004; 329: 881 .

7 Ganguli M, Du Y, Dodge HH, Ratcliff GG, Chang CC. Depressive symptoms and cognitive decline in late life: a prospective epidemiological study. Arch Gen Psychiatry 2006; 63: 153-60. 
8 Ownby RL, Crocco E, Acevedo A, John V, Loewenstein D. Depression and risk for Alzheimer disease: systematic review, meta-analysis, and metaregression analysis. Arch Gen Psychiatry 2006; 63: 530-8.

9 Woodward M, Oliphant J, Lowe G, Tunstall-Pedoe H. Contribution of contemporaneous risk factors to social inequality in coronary heart disease and all causes mortality. Prev Med 2003; 36: 561-8.

10 Wilson KC, Chen R, Taylor S, McCracken CF, Copeland JR. Socio-economic deprivation and the prevalence and prediction of depression in older community residents. The MRC-ALPHA Study. Br J Psychiatry 1999; 175: 549-53.

11 Almeida OP, Flicker L, Norman P, Hankey GJ, Vasikaran S, van Bockxmeer FM, Jamrozik K. Association of Cardiovascular Risk Factors and Disease With Depression in Later Life. Am J Geriatr Psychiatry 2006; 15: 506-13.

12 Chen R, Wei L, Hu Z, Qin X, Copeland JR, Hemingway H. Depression in older people in rural China. Arch Intern Med 2005; 165: 2019-25.

13 Chen R, Copeland JR, Wei L. A meta-analysis of epidemiological studies in depression of older people in the People's Republic of China. Int J Geriatr Psychiatry 1999; 14: 821-30.

14 Chen R, Hu Z, Qin X, Xu X, Copeland JR. A community-based study of depression in older people in Hefei, China - the GMS-AGECAT prevalence, case validation and socio-economic correlates. Int J Geriatr Psychiatry 2004; 19: $407-13$.

15 Hu FB, Wang B, Chen C, Jin Y, Yang J, Stampfer MJ, Xu X. Body mass index and cardiovascular risk factors in a rural Chinese population. Am J Epidemiol 2000; 151: 88-97.

16 Chen Z, Peto R, Collins R, MacMahon S, Lu J, Li W. Serum cholesterol concentration and coronary heart disease in population with low cholesterol concentrations. BMJ 1991; 303: 276-82.

17 Copeland JR, Prince M, Wilson KC, Dewey ME, Payne J, Gurland B. The Geriatric Mental State Examination in the 21st century. Int J Geriatr Psychiatry 2002; 17: 729-32.

18 Copeland JR, McCracken CF, Dewey ME, Wilson KC, Doran M, Gilmore C, Scott A, Larkin BA. Undifferentiated dementia, Alzheimer's disease and vascular dementia: age- and gender-related incidence in Liverpool. The MRC-ALPHA Study. Br J Psychiatry 1999; 175: 433-8.

19 Saunders PA, Copeland JR, Dewey ME, Davidson IA, McWilliam C, Sharma V Sullivan $\mathrm{Cl}$. Heavy drinking as a risk factor for depression and dementia in elderly men. Findings from the Liverpool longitudinal community study. Br J Psychiatry 1991; 159: 213-6.

20 Copeland JRM, Dewey ME, Griffiths-Jones HM. Dementia and depression in elderly persons: AGECAT compared with DSMII and pervasive illness. Int $J$ Geriatr Psychiatry 1990; 5: 47-51.

21 Copeland JR, Chen R, Dewey ME, McCracken CF, Gilmore C, Larkin B, Wilson $\mathrm{KC}$. Community-based case-control study of depression in older people. Cases and sub-cases from the MRC-ALPHA Study. Br J Psychiatry 1999; 175 $340-7$.

22 Liu J, Li S, Zhang WX, Chen CH. Assessment of computerized diagnostic system of Geriatric Mental State schedule shortened community version (GMS-AGECAT). China Psychol Health J 2001; 15: 220-2.

23 Kua EH. A community study of mental disorders in elderly Singaporean Chinese using the GMS-AGECAT package. Aust N Z J Psychiatry 1992; 26: 502-6.

24 American Psychiatric Association. Diagnostic and Statistical Manual of Mental Disorders (3rd edn) (DSM-III). APA, 1980.

25 American Psychiatric Association. Diagnostic and Statistical Manual of Mental Disorders (4th edn) (DSM-IV). APA, 1980.

26 Altman DG, Bland JM. Interaction revisited: the difference between two estimates. BMJ 2003; 326: 219.

27 Townsend P, Phillimore $\mathrm{P}$, Beattie A. Health and Deprivation: Inequality and the North. Croom Helm, 1988.

28 Wilson KC, Copeland JR, Taylor S, Donoghue J, McCracken CF. Natural history of pharmacotherapy of older depressed community residents. The MRC-ALPHA Study. Br J Psychiatry 1999; 175: 439-43.

29 Prince $M$, Acosta $D$, Chiu $H$, Scazufca $M$, Varghese M. Dementia diagnosis in developing countries: a cross-cultural validation study. Lancet 2003; 361 909-17.

30 Prince $M$, Acosta D, Chiu H, Copeland J, Dewey M, Scazufca M, Varghese M; 10/66 Dementia Research Group. Effects of education and culture on the validity of the Geriatric Mental State and its AGECAT algorithm. Br J Psychiatry 2004; 185: 429-36.

31 Chen R, Hu Z, Wei L, Qin X, Copeland JR. I. The relationship between syndromes of depression and dementia temporal? The MRC-ALPHA and Hefei-China studies. Psychol Med 2008; 23: 1-6.

\section{Attachment theory and the psychiatrist-patient relationship}

\section{Jeremy Holmes}

When subject to stress, threat or illness, humans seek an older wiser figure or 'secure base'. The patient comes to the psychiatrist in a state of arousal. An empathic response on the part of the doctor, with accurate verbal identification of emotion, produces assuagement of attachment behaviours, triggering 'vitality affects' and the beginnings of 'companionable exploration' - the reasons for coming and history of presenting symptoms. People with insecure attachment histories find this process problematic, typically 'deactivating' or 'hyperactivating' affect, or producing incoherence. Psychiatrists need to identify and understand this sequence of relational expectations and behaviours in themselves and their clients. 